OPEN ACCESS

Edited by:

Patrick Andrew Thompson, Baylor College of Medicine,

United States

Reviewed by:

Stuart MacLeod,

University of British Columbia, Canada

Ashwin Karanam,

Pfizer, United States

${ }^{*}$ Correspondence:

Xiaowen Zhai

zhaixiaowendy@163.com

Hong Xu

hxu@shmu.edu.cn

Zhiping $\mathrm{Li}$

zpli@fudan.edu.cn

${ }^{+}$These authors have contributed equally to this work

Specialty section:

This article was submitted to Obstetric and Pediatric Pharmacology, a section of the journal

Frontiers in Pharmacology

Received: 17 February 2021

Accepted: 26 October 2021

Published: 08 November 2021

Citation:

Chen $X$, Wang $D$, Wang G, Huang $Y$, Yu X, Lu J, Zhai X, Xu H and LiZ (2021) Optimization of Initial Dose Regimen for

Sirolimus in Pediatric Patients With Lymphangioma.

Front. Pharmacol. 12:668952.

doi: 10.3389/fphar.2021.668952

\section{Optimization of Initial Dose Regimen for Sirolimus in Pediatric Patients With Lymphangioma}

\author{
Xiao Chen ${ }^{1 \dagger}$, Dongdong Wang ${ }^{1 \dagger}$, Guangfei Wang ${ }^{1 \dagger}$, Yidie Huang ${ }^{1}, X_{i n} \mathrm{Yu}^{1}$, Jinmiao $\mathrm{Lu}^{1}$, \\ Xiaowen $\mathrm{Zhai}^{2 *}$, Hong $\mathrm{Xu}^{3 *}$ and Zhiping $\mathrm{Li}^{1 *}$ \\ ${ }^{1}$ Department of Pharmacy, National Children's Medical Center, Children's Hospital of Fudan University, Shanghai, China, \\ ${ }^{2}$ Department of Hematology and Oncology, National Children's Medical Center, Children's Hospital of Fudan University, Shanghai, \\ China, ${ }^{3}$ Department of Nephrology, National Children's Medical Center, Children's Hospital of Fudan University, Shanghai, China
}

Sirolimus is an effective oral treatment for pediatric patients with lymphangioma. The present clinical study in 15 children (0.12-16.39 years of age) examines the effects of underlying factors on sirolimus concentrations through application of a population pharmacokinetic model. Using Monte Carlo simulation, an initial dose regimen for sirolimus in pediatric patients with lymphangioma is presented. It is found that the lower the body weight, the higher the clearance rate and sirolimus clearances are $0.31-0.17 \mathrm{~L} / \mathrm{h} / \mathrm{kg}$ in pediatric patients with lymphangioma whose weights are $5-60 \mathrm{~kg}$, respectively. The doses of sirolimus, $0.07,0.06,0.05 \mathrm{mg} / \mathrm{kg} / \mathrm{day}$ are recommended for weights of $5-10,10-24.5$ and $24.5-60 \mathrm{~kg}$ in children with lymphangioma. This study is the first to establish a population pharmacokinetic model for sirolimus and to recommend initial doses in pediatric patients with lymphangioma. Large scale, prospective studies are needed in the future.

Keywords: optimization, initial dose regimen, sirolimus, pediatric, lymphangioma

\section{INTRODUCTION}

Lymphangioma, which is also called lymphatic malformation, is rare and benign anomaly derived from the defective embryological development of the primordial lymphatic structure (Amodeo et al., 2017b). Disease progress includes the progressive growth of the lymphangioma leading to compression of the adjacent structures (Reinglas et al., 2011). On account of their permeative growth throughout all tissue layers, treatment is often challenging and controversial (Laforgia et al., 2016; Amodeo et al., 2017b). Historically, surgical excision is one choice of treatment considered; however, less invasive therapy is now preferred (Amodeo et al., 2017b).

At present, it has been reported that sirolimus is an oral effective medication for lymphangioma (Reinglas et al., 2011; Amodeo et al., 2017a; Amodeo et al., 2017b). However, sirolimus has a narrow therapeutic window and its considerable inter- and intra-individual pharmacokinetic variabilities make it difficult to design an initial dosing schedule (Chen et al., 2020; Cheng et al., 2020; Wang et al., 2020), especially in pediatric patients with lymphangioma. Hence, the present study aims to investigate the effects of underlying factors on clinical sirolimus concentrations by building up a population pharmacokinetic model, and to recommend initial dose regimen for sirolimus in pediatric patients with lymphangioma using Monte Carlo simulation. 


\section{METHODS}

\section{Patients}

Pediatric patients with lymphangioma from May 2016 to December 2020 at the Children's Hospital of Fudan University (Shanghai, China) were collected, retrospectively. The study was approved by the Research Ethics Committee of Children's Hospital of Fudan University [Ethical code: (2019) 019]. Because the study was retrospective, it was approved by the ethics committee of our hospital without the need for written informed consent. The inclusion criteria were 1) treated with sirolimus, and 2) recorded therapeutic drug monitoring (TDM) for sirolimus. Demographic data of patients were collected, such as gender, age, weight, albumin, alanine transaminase, aspartate transaminase, creatinine, urea, total protein, total bile acid, direct bilirubin, total bilibrubin, hematocrit, hemoglobin, mean corpuscular hemoglobin, mean corpuscular hemoglobin concentration.

\section{TDM}

The Emit 2000 Sirolimus Assay (Siemens Healthcare Diagnostics Inc.) with range of linear response, 3.5-30 ng/ $\mathrm{ml}$, whose values of inter-assay variability [coefficient of variation $(\mathrm{CV} \%)]<4.0 \%$, and values of intra-assay $\mathrm{CV}(\%)$ $<6.2 \%$, were used to measure sirolimus concentrations.

\section{Population Pharmacokinetic Model}

The non-linear mixed-effects modeling software, NONMEM (edition 7, ICON Development Solutions, Ellicott City, MD, USA) and a first-order conditional estimation method with interaction (FOCE-I method) were used to establish the population pharmacokinetic model of sirolimus in pediatric patients with lymphangioma. The apparent oral clearance $(\mathrm{CL} / \mathrm{F})$, volume of distribution $(\mathrm{V} / \mathrm{F})$, and absorption rate constant, $\mathrm{Ka}$ (fixed at $0.485 / \mathrm{h}$ ), were included in pharmacokinetic parameters (Wang et al., 2020).

\section{Random Effect Model}

Equation 1 showed the inter-individual variability:

$$
\mathrm{M}_{\mathrm{i}}=\mathrm{TV}(\mathrm{M}) \times \exp \left(\eta_{i}\right)
$$

$\mathrm{M}_{\mathrm{i}}$, the individual parameter value; $\mathrm{TV}(\mathrm{M})$, the typical individual parameter value; $\eta_{i}$, symmetrical distribution, which was random term with zero mean and variance omega $2\left(\omega^{2}\right)$.

Equation 2 showed random residual variability

$$
\mathrm{N}_{\mathrm{i}}=\mathrm{L}_{\mathrm{i}}+\varepsilon_{1}
$$

$\mathrm{N}_{\mathrm{i}}$, the observed concentration; $\mathrm{L}_{\mathrm{i}}$, the individual predicted concentration; $\varepsilon_{1}$, symmetrical distribution, which was random term with zero mean and variance sigmâ2 $\left(\sigma^{2}\right)$.

\section{Covariate Model}

Equation 3 showed the relations of pharmacokinetic parameters with weight:

$$
\mathrm{X}_{\mathrm{i}}=\mathrm{X}_{\text {std }} \times\left(\mathrm{Y}_{\mathrm{i}} / \mathrm{Y}_{\text {std }}\right)^{\text {power }}
$$

$\mathrm{X}_{\mathrm{i}}$, the $i$ th individual parameter; $\mathrm{Y}_{\mathrm{i}}$, the $i$ th individual weight; $\mathrm{Y}_{\text {std }}$, the standard weight of $70 \mathrm{~kg}$; $\mathrm{X}_{\text {std }}$, the typical individual parameter, whose weight was $\mathrm{Y}_{\text {std }}$; power, the allometric coefficient: 0.75 for the $\mathrm{CL} / \mathrm{F}$ and 1 for the $\mathrm{V} / \mathrm{F}$ (Anderson and Holford, 2008).

Equation 4 and Equation 5 showed continuous or categorical covariates with pharmacokinetic parameters, respectively:

$$
\begin{gathered}
\mathrm{Z}_{\mathrm{i}}=\operatorname{TV}(Z) \times\left(\operatorname{Cov}_{\mathrm{i}} / \operatorname{Cov}_{\text {median }}\right)^{\theta} \\
\mathrm{Z}_{\mathrm{i}}=\mathrm{TV}(\mathrm{Z}) \times \theta^{\mathrm{Covi}}
\end{gathered}
$$

$Z_{i}$, the individual parameter value; $T V(Z)$, the typical individual parameter value; $\theta$, the parameter to be estimated; $\mathrm{Cov}_{\mathrm{i}}$, the covariate of the $i$ th individual; $\operatorname{Cov}_{\text {median }}$, the population median for the covariate.

Objective function value (OFV) changes were used as the covariate inclusion criteria, whose decrease greater than $3.84(p<$ $0.05)$ was considered sufficient for inclusion in the base model. An increase greater than $6.63(p<0.01)$ was considered sufficient for significance in the final model.

\section{Model Evaluation}

The goodness-of-fit plots of model, conditional weighted residuals (WRES) vs. time after the start therapy, observations $v s$. population predictions, observations vs. individual predictions, conditional WRES $v s$. population predictions, conditional WRES vs. individual predictions were used to evaluate model visualization. Distribution of weighted residuals for model, density vs. weighted residuals, and quantilies of weighted residuals $v s$. quantilies of normal, were used to evaluate the model distribution. Prediction-corrected visual predictive check (VPC) of model were used to evaluate model predictability. Additionally, 1,000 time bootstraps were used to evaluate model stability.

\section{Simulation}

The target concentration window of sirolimus in pediatric patients with lymphangioma was $5-15 \mathrm{ng} / \mathrm{ml}$ (Reinglas et al., 2011; Amodeo et al., 2017b; Lagreze et al., 2019), and initial doses were simulated using Monte Carlo method. In the present study, 1,000 virtual pediatric patients were simulated in seven weight groups $(5,10,20,30,40,50$, and $60 \mathrm{~kg})$ for ten dosages $(0.01,0.02,0.03,0.04,0.05,0.06,0.07,0.08,0.09$, and $0.10 \mathrm{mg} / \mathrm{kg} /$ day), respectively. The sirolimus regimen was divided evenly into two doses per day. The evaluation criteria were the probabilities of achieving concentrations within the target window.

\section{RESULTS}

\section{Patients}

A total of 15 pediatric patients with lymphangioma are included in the present study, 12 boys and 3 girls, aged from 0.12 to 16.39 years old and weighted from 4 to $54 \mathrm{~kg}$. Demographic data of pediatric patients with lymphangioma are shown in Table 1. 
TABLE 1 | Demographic data of patients.

\begin{tabular}{|c|c|c|}
\hline Characteristic & Mean \pm SD & Median (range) \\
\hline Gender (boys/girls) & $12 / 3$ & - \\
\hline Age (years) & $7.29 \pm 3.11$ & $6.80(0.12-16.39)$ \\
\hline Weight ( kg) & $22.27 \pm 9.87$ & $21.00(4.00-54.00)$ \\
\hline Albumin ( $\mathrm{g} / \mathrm{L})$ & $37.82 \pm 7.52$ & $38.50(21.60-46.40)$ \\
\hline Alanine transaminase ( IU/L) & $22.41 \pm 23.33$ & $15.45(6.00-83.60)$ \\
\hline Aspartate transaminase ( IU/L) & $40.61 \pm 32.19$ & $36.00(16.00-133.80)$ \\
\hline Creatinine ( $\mu \mathrm{mol} / \mathrm{L})$ & $32.36 \pm 16.18$ & $29.00(16.00-76.00)$ \\
\hline Urea ( mmol/L) & $3.89 \pm 1.18$ & $3.70(2.20-5.70)$ \\
\hline Total protein ( g/L) & $63.88 \pm 15.90$ & 68.50 (30.80-91.90) \\
\hline Total bile acid ( $\mu \mathrm{mol} / \mathrm{L})$ & $5.03 \pm 2.63$ & $5.30(1.20-10.30)$ \\
\hline Direct bilirubin ( $\mu \mathrm{mol} / \mathrm{L})$ & $1.63 \pm 0.86$ & $1.60(0.60-3.00)$ \\
\hline Total bilibrubin ( $\mu \mathrm{mol} / \mathrm{L})$ & $4.77 \pm 2.22$ & $4.40(1.80-9.40)$ \\
\hline Hematocrit (\%) & $33.19 \pm 5.26$ & $32.40(23.00-43.40)$ \\
\hline Hemoglobin ( $\mathrm{g} / \mathrm{L})$ & $109.26 \pm 20.99$ & $104.00(77.00-156.00)$ \\
\hline Mean corpuscular hemoglobin (pg) & $26.34 \pm 2.58$ & $26.00(22.00-31.00)$ \\
\hline Mean corpuscular hemoglobin concentration ( $\mathrm{g} / \mathrm{L}$ ) & $328.00 \pm 16.27$ & $331.00(292.00-359.00)$ \\
\hline
\end{tabular}

\section{Modeling}

In the final model, weight is included as a covariant and the final model is shown below:

$$
\begin{gathered}
\mathrm{CL} / \mathrm{F}=11.3 \times(\text { weight } / 70)^{0.75} \\
\mathrm{~V} / \mathrm{F}=388 \times(\text { Weight } / 70)
\end{gathered}
$$

$\mathrm{CL} / \mathrm{F}$, apparent oral clearance; V/F, apparent volume of distribution.

\section{Evaluation}

Time-concentration diagrams and model evaluations are shown in Figure 1. Figure 1A displays observations $v s$.time after the start therapy, and Figures 1B-F show goodness-of-fit plots of model, in which a good model fitting effect is demonstrated. Figures 2A,B demonstrate the distribution of weighted residuals, following the normal distribution. Figure 2C shows predictioncorrected VPC on the model confirming that most of the observed sirolimus concentrations are within the $95 \%$ prediction intervals from the simulation data, and the prediction-corrected sirolimus concentrations are well predicted by the final model. In Table 2, the parameter estimates of the final model are within 95\% confidence interval of 1,000 bootstraps, showing that the model is reliable.

\section{Simulation}

Figure 2D displays the clearance rate of sirolimus in pediatric patients with lymphangioma, and we find that the lower the body weight, the higher the clearance rate and sirolimus clearances are $0.31-0.17 \mathrm{~L} / \mathrm{h} / \mathrm{kg}$ in pediatric patients with lymphangioma, whose weight are 5-60 kg, respectively. Initial dose simulations are shown in Figure 3, and Figures $\mathbf{3 A} \mathbf{A} \mathbf{J}$ are for ten dosages $(0.01,0.02,0.03,0.04,0.05,0.06,0.07,0.08,0.09$, and $0.10 \mathrm{mg} / \mathrm{kg} /$ day), respectively. The evaluation criteria are the probabilities of achieving target concentration window, and the doses of sirolimus, $0.07,0.06,0.05 \mathrm{mg} / \mathrm{kg} / \mathrm{day}$ are recommended for weights of 5-10, 10-24.5, and $24.5-60 \mathrm{~kg}$ in pediatric patients with lymphangioma, respectively, which are shown in Figure 4 and Table 3. In addition, Figure 5 shows the security aspect and at the current recommended doses, the probabilities to exceed the upper limit of the target concentrations are less than $5 \%$, indicating relatively high security.

\section{DISCUSSION}

Sirolimus, the mammalian target of rapamycin (mTOR) inhibitor, is an immunosuppressive and antitumor drug. The mTOR signaling is now known to be linked to vascular development, whose activation can increase the expression of vascular endothelial growth factor $\mathrm{A}$ and $\mathrm{C}$, crucial regulators of angiogenesis and lymphangiogenesis respectively, promoting vascular proliferation and cell growth (Amodeo et al., 2017b). Sirolimus can block the mTOR signaling and further inhibit abnormal vascular proliferation, reducing the production of vascular endothelial growth factor and responsiveness of its receptors (Neuhaus et al., 2001; Huber et al., 2007; Kobayashi et al., 2007; Lee and Hung, 2007; Hammill et al., 2011; Shibuya, 2011).

Sirolimus was first used as an immunosuppressant agent to augment prevention of rejection in solid organ transplantation, following kidney transplant (Pinto-Leite et al., 2009). However, at present, many studies have reported that sirolimus can be used as an anticancer treatment (Wan and Helman, 2007; Cloughesy et al., 2008). For example, sirolimus is a potentially effective treatment option for patients with hormone receptor-positive advanced breast cancer (Yi et al., 2020). The combination of pemetrexed and sirolimus is active in previously treated nonsmall cell lung cancer (Komiya et al., 2019). Combination of metformin and sirolimus show the most potent inhibition in all colon cancer cell lines (Mussin et al., 2017). Switching from calcineurin inhibitors to sirolimus has an antitumoral effect among kidney-transplant recipients with previous squamouscell carcinoma (Euvrard et al., 2012). In addition, it has been also reported that sirolimus is an oral effective medication for 


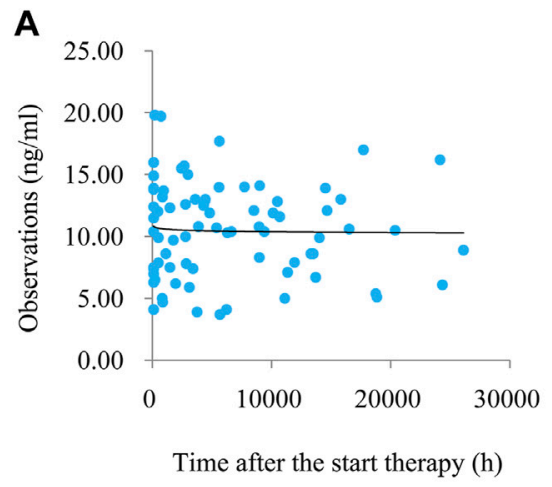

C

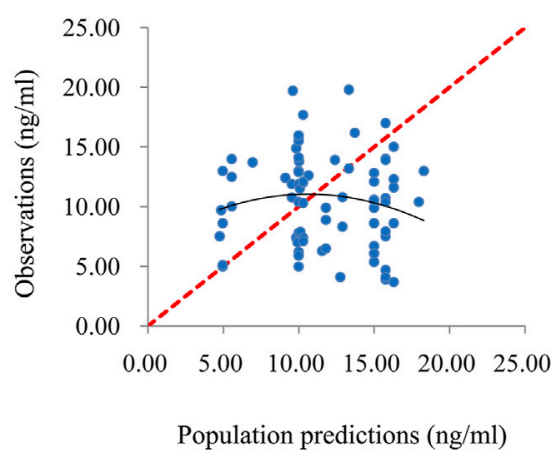

E

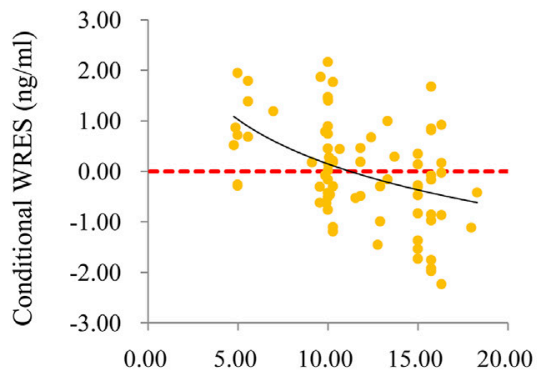

Population predictions (ng/ml)

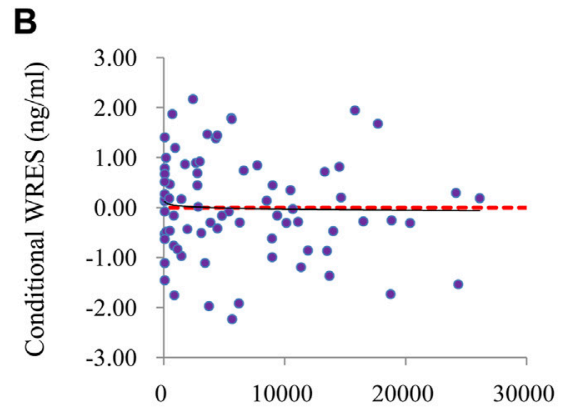

Time after the start therapy (h)

\section{D}

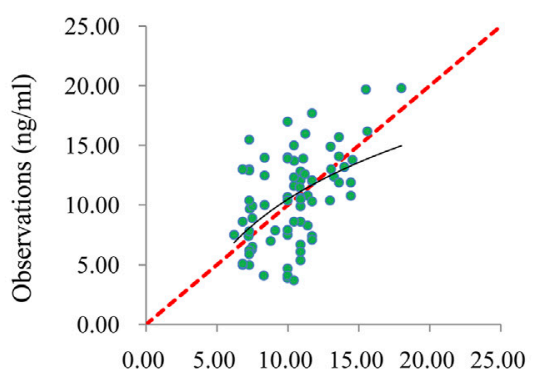

Individual predictions ( $\mathrm{ng} / \mathrm{ml})$

$\mathbf{F}$

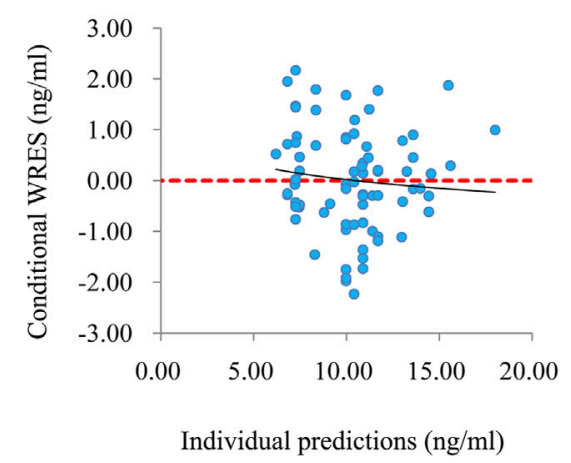

FIGURE 1 | The goodness-of-fit plots of model. (A) Time-concentration diagram. (B) Conditional weighted residuals (WRES) vs. time after the start therapy. (C) Observations vs. population predictions. (D) Observations vs. individual predictions. (E) Conditional WRES vs. population predictions. (F) Conditional WRES vs. individual predictions.

lymphangioma (Reinglas et al., 2011; Amodeo et al., 2017a; Amodeo et al., 2017b).

Lymphangioma is an uncommon, benign malformations of the lymphatic system that can occur anywhere on the skin and mucous membranes (Miceli and Stewart, 2020). Lymphangioma can be categorized as deep or superficial based on the depth and size of the abnormal lymphatic vessels or as congenital or acquired (Miceli and Stewart, 2020). In recent years there have been increasing reports on the treatment of lymphangioma with sirolimus (Amodeo et al., 2017b; Lagreze et al., 2019). However, the adverse effects of sirolimus have limited its clinical application to some extent. Its most significant adverse effects are anemia, anorexia, asthenia, edema, elevated alkaline phosphatase, elevated serum creatinine, hyperglycemia, hyperlipemia, hypertriglyceridemia, hypophosphatemia, lymphopenia, nausea, rash, thrombocytopenia (Amodeo et al., 2017b). In addition, rare but potentially serious adverse effects of sirolimus have also been found such as cardiac disorders and infections (Klumpen et al., 2010; Ji et al., 2017).

The majority of adverse effects are related to the concentrations of sirolimus, and in clinical practice, TDM is used to measure sirolimus concentrations. The following doses of sirolimus are regulated on the strength of concentrations. However, with narrow therapeutic range and $\mathrm{PK}$ variabilities it is hard to project an initial dosing schedule for use in pediatric patients with lymphangioma. Fortunately, the 
A

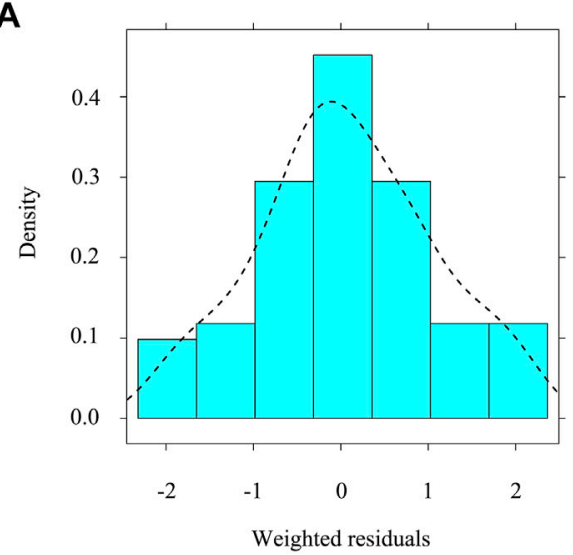

C

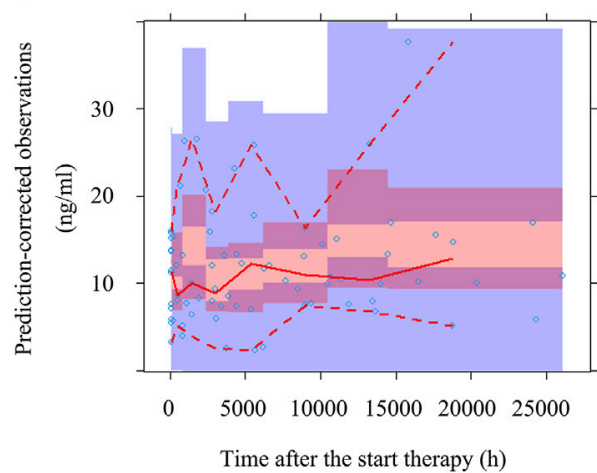

B

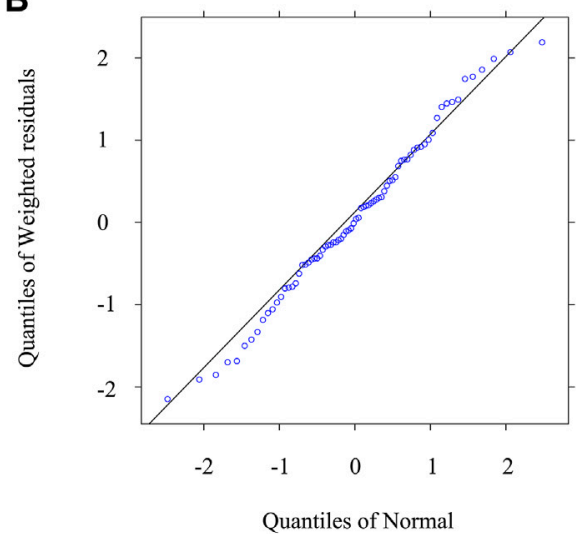

D

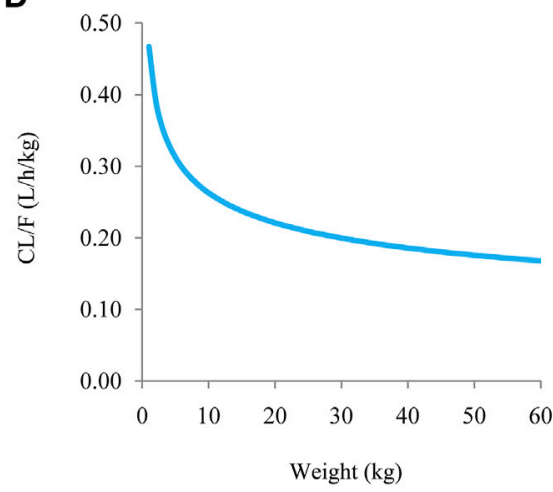

FIGURE 2 | The evaluation of normality and VPC. (A) Density vs. weighted residuals. (B) Quantilies of weighted residuals vs. quantilies of normal. (C) Predictioncorrected visual predictive check (VPC) of model. The middle solid line represents the median of the prediction-corrected concentrations. The lower and upper dashed lines are the 2.5 and 97.5 th percentiles of the prediction-corrected concentrations. (D) Sirolimus apparent clearance rate of pediatric patients with different weight.

TABLE 2 | Parameter estimates of final model and bootstrap validation.

\begin{tabular}{|c|c|c|c|}
\hline \multirow[t]{2}{*}{ Parameter } & \multirow[t]{2}{*}{ Estimate } & \multirow[t]{2}{*}{ SE (\%) } & $\begin{array}{l}\text { Bootstrap } \\
(n=1,000)\end{array}$ \\
\hline & & & $95 \%$ confidence interval \\
\hline CL/F (L/h/70 kg) & 11.3 & 9.5 & $(0.7,13.4)$ \\
\hline V/F (L/70 kg) & 388 & 41.8 & $(1,648)$ \\
\hline $\mathrm{Ka}\left(\mathrm{h}^{-1}\right)$ & 0.485 (fixed) & - & - \\
\hline$\omega \mathrm{CL} / \mathrm{F}$ & $0.303(13.7 \%)$ & 18.5 & $(0.102,0.355)$ \\
\hline$\sigma 1(\mathrm{ng} / \mathrm{ml})$ & 3.578 & 3.7 & (3.056, 3.808) \\
\hline
\end{tabular}

95\% confidential interval was displayed as the 2.5 and 97.5 th percentile of bootstrap estimates. CL/F, apparent oral clearance (L/h); V/F, apparent volume of distribution (L); Ka, absorption rate constant $\left(h^{-1}\right)$; $\omega_{C L / F}$ inter-individual variability of $\mathrm{CL} / F$; $\sigma_{1}$, sigma, residual variability, additive error.

optimum initial regimen can be recommended by the combination of population pharmacokinetics and Monte Carlo simulation. For example, Cheng et al reported the first study in pediatric: population pharmacokinetics of sirolimus and its application in Chinese children with immune cytopenia (Cheng et al., 2020). Wang et al reported initial dosage recommendation for sirolimus in children with tuberous sclerosis complex (Wang et al., 2020). Chen et al reported initial dose recommendation for sirolimus in paediatric kaposiform haemangioendothelioma patients (Chen et al., 2020).
Thus, our study aims to explore the effects of underlying factors on clinical sirolimus concentrations with a population pharmacokinetic model, and to recommend initial dose regimen for sirolimus in pediatric patients with lymphangioma.

In the present study, sirolimus clearance is affected by weight. It had been reported that there is a non-linear relationship between drug clearance and weight in pediatric patients, which may be well described by allometric scaling with the coefficients of 0.75 for clearance and 1 for volume (Anderson and Holford, 2008; Hao et al., 2018). In addition, we find that the lower the body weight, the higher the clearance rate. In other words, the smaller the child's weight, the higher the dose required. Afterwards, the initial dosing protocol is simulated based on the final model, the target concentration window of sirolimus in pediatric patients with lymphangioma is 5-15 ng/ml (Reinglas et al., 2011; Amodeo et al., 2017b; Lagreze et al., 2019). The doses of sirolimus, 0.07, 0.06, and $0.05 \mathrm{mg} / \mathrm{kg}$ are recommended respectively for weights of 5-10, 10-24.5, and $24.5-60 \mathrm{~kg}$ in pediatric patients with lymphangioma. "Children are NOT small adults", and our innovation lies in that the model we use to simulate the dose is taken from the population of pediatric patients with 


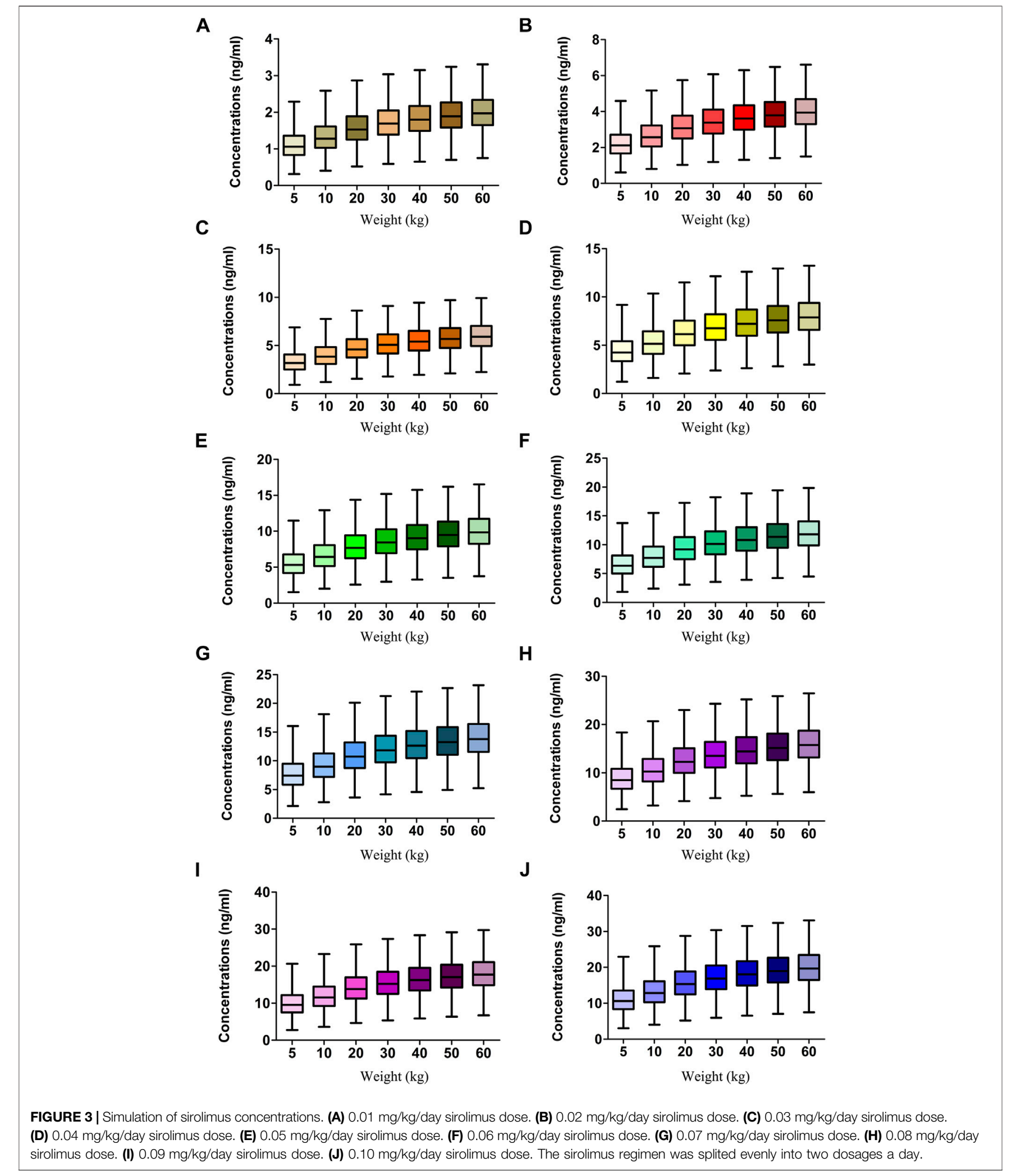

lymphangioma, so that the model can more accurately reflect the real situation of sirolimus in pediatric patients with lymphangioma, instead of referring to adult doses again.
The metabolism of sirolimus has been shown to be influenced by CYP3A4 and CYP3A5 in adults (Tamashiro et al., 2017; Zhang et al., 2017). However, no significant effect such as this was found in 


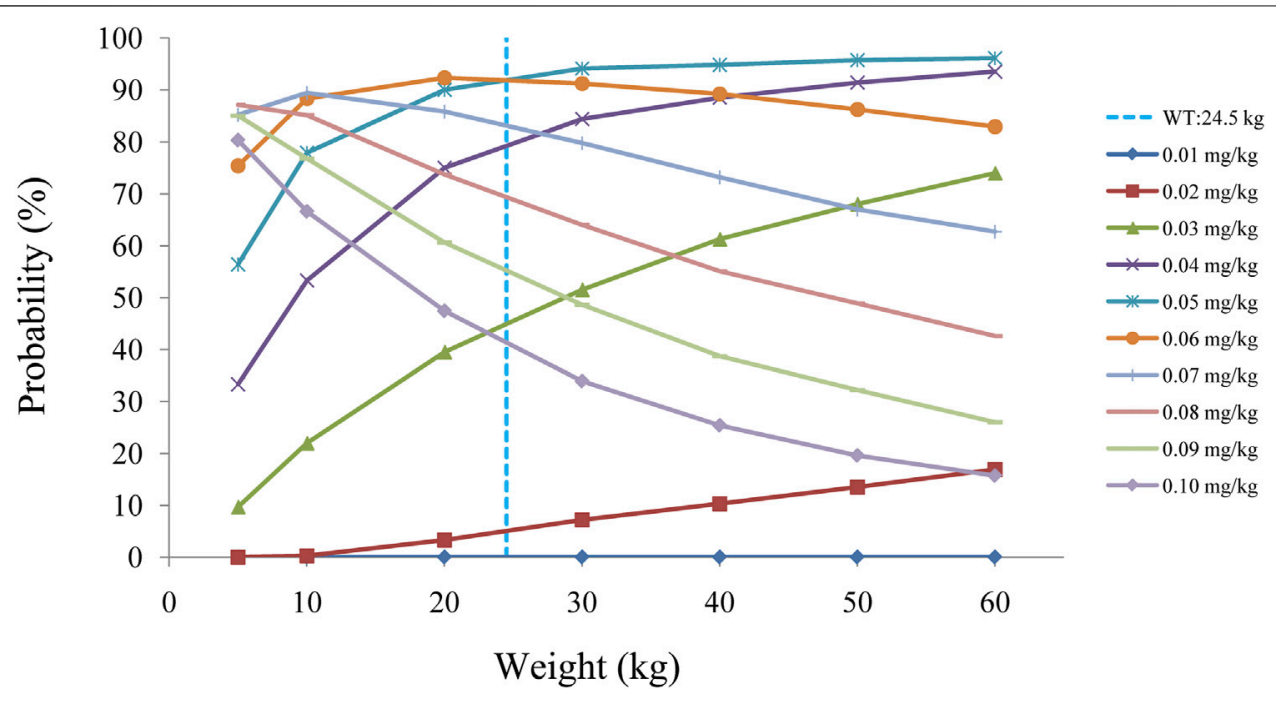

FIGURE 4 | Probability to achieve the target concentrations.

TABLE 3 | Initial dosage recommendation of sirolimus.

Body weight ( kg)

Dose (mg/kg/day) ${ }^{a}$
Probability to achieve the target concentrations

(\%)
Probability to exceed the upper limit

of the target concentrations (\%)

$\begin{array}{ll}5-10 & 0.07 \\ 10-24.5 & 0.06 \\ 24.5-60 & 0.05\end{array}$

5-10

$24.5-60$

${ }^{a}$ Split evenly into two doses a day.

$85.2-89.4$
$88.3-92.3$
$91.1-96.1$

$85.2-89.4$

$91.1-96.1$
$1.0-4.7$

$0.3-4.91$

$0.045-2.6$

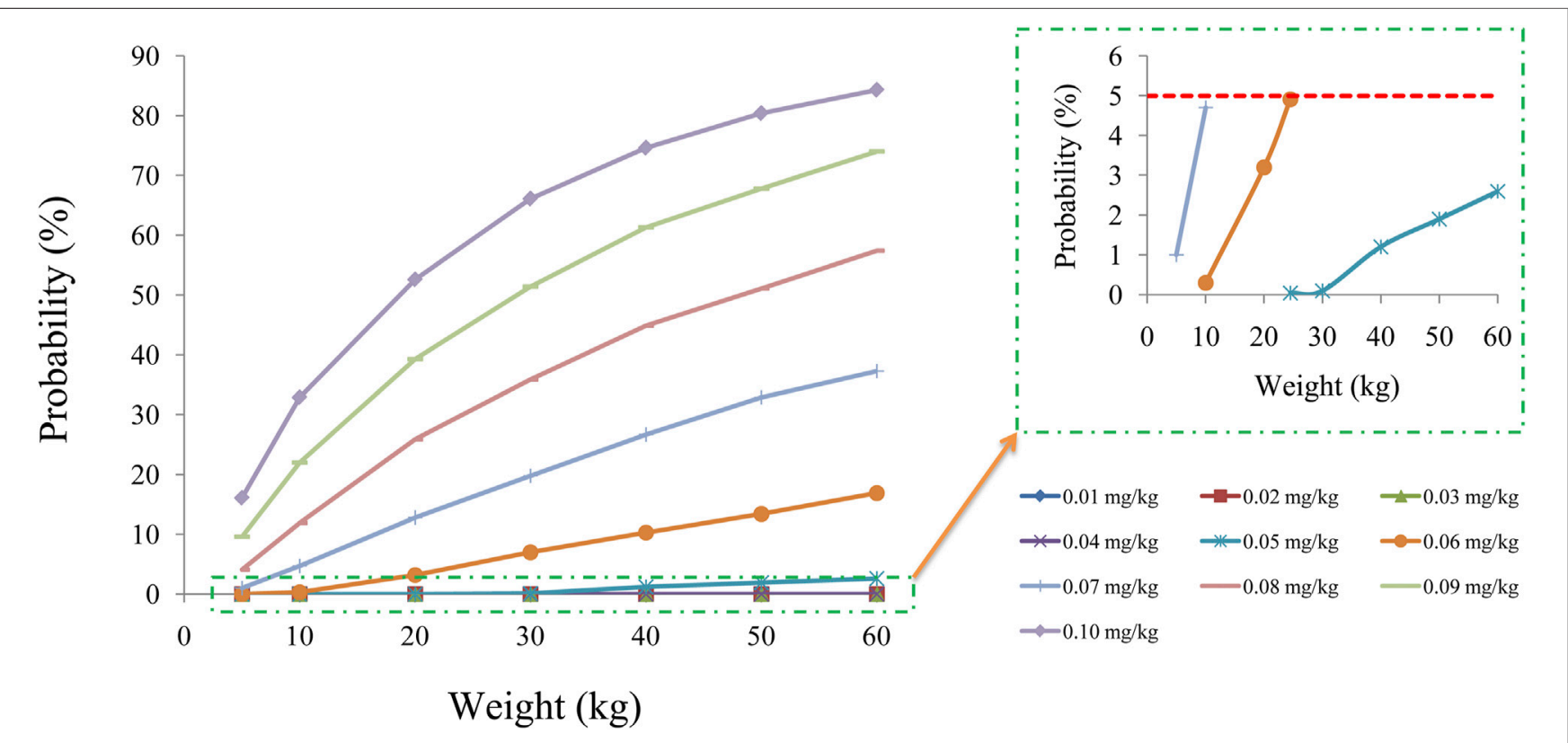

FIGURE $\mathbf{5}$ | Probability to exceed the upper limit of the target concentrations. 
children. For example, CYP3A4 and CYP3A5 polymorphism did not significantly affect sirolimus clearance rates in children with tuberous sclerosis complex (Wang et al., 2020). Also, CYP3A genotypes were not included in population pharmacokinetics of sirolimus for children with recurrent solid tumours or complicated vascular anomalies (Mizuno et al., 2017a; Mizuno et al., 2017b). Thus, our study do not further explore the impacts from CYP3A4 and CYP3A5 genotypes. Furthermore, no routine tests are performed for sirolimus pharmacogenomics in clinical therapy. The dose recommendation on body weight was considered to be more convenient and practical in a clinical setting (Wang et al., 2020). And there are evidences to support this conclusion. For example, in Wang et als study, only body weight was fixed in the covariate model (Wang et al., 2020). And in other drug studies, such as in Niu et als study, population pharmacokinetics and dosing regimen optimisation of lopinavir in Chinese adults infected with HIV, only body weight was included into model (Niu et al., 2019).

However, due to the low incidence of lymphangioma, the present study has objective difficulties in the collection of children patients, resulting in the limited number of pediatric patients with lymphangioma. A total of 15 pediatric patients with lymphangioma are included in the present study. Given the low incidence of the disease, our small number of patients may be objectively acceptable. Because similar studies with smaller numbers have been reported, for example, in Mizuno et al's study, total 19 patients were included (Mizuno et al., 2017a). In addition, in Wang et als study, total 15 patients were included (Wang et al., 2020). Meanwhile, similarly, in Mizuno et als study, the covariate analysis inclusion of age and sex did not significantly improve the temsirolimus model (Mizuno et al., 2017a). In Wang et al's study, only body weight was fixed in the covariate model, and recommending doses were based on body weight (Wang et al., 2020). As for the limited sample size, these covariates should still be evaluated in an adequately powered future study. But then again, in the current limited number of children, body weight as a covariable can better fit the clinical data in the real world, which to some extent can provide beneficial reference for solving the missing individual drug administration of sirolimus in pediatric patients with lymphangioma, and better lay the foundation for the following large-scale clinical research. Even so, studies with larger sample sizes need to be further studied in the future.

\section{CONCLUSION}

This study has limitations because of the rarity of lymphangioma and the use of retrospective observations. However, it is the first

\section{REFERENCES}

Amodeo, I., Cavallaro, G., Raffaeli, G., Colombo, L., Fumagalli, M., Cavalli, R., et al. (2017a). Abdominal Cystic Lymphangioma in a Term Newborn: A Case Report and Update of New Treatments. Medicine (Baltimore) 96 (8), e5984. doi:10.1097/MD.0000000000005984

Amodeo, I., Colnaghi, M., Raffaeli, G., Cavallaro, G., Ciralli, F., Gangi, S., et al. (2017b). The Use of Sirolimus in the Treatment of Giant Cystic Lymphangioma: Four Case Reports and Update of Medical Therapy. Medicine (Baltimore) 96 (51), e8871. doi:10.1097/MD.0000000000008871 report of a population pharmacokinetic model for sirolimus with recommendations for initial dosing in pediatric patients with lymphangioma. Large scale and prospective studies are needed in the future to confirm and refine the preliminary findings presented here.

\section{DATA AVAILABILITY STATEMENT}

The raw data supporting the conclusions of this article will be made available by the authors, without undue reservation.

\section{ETHICS STATEMENT}

The studies involving human participants were reviewed and approved by Research Ethics Committee of Children's Hospital of Fudan University. Written informed consent from the participants' legal guardian/next of kin was not required to participate in this study in accordance with the national legislation and the institutional requirements.

\section{AUTHOR CONTRIBUTIONS}

$\mathrm{XC}$ and DW designed the study, collected the data, analyzed the data, visualized the data and drafted the manuscript. GW, YH, $\mathrm{XY}$, and JL collected the data. XZ, HX and ZL designed the study and acquired funding.

\section{FUNDING}

This work was supported by the Shanghai Municipal Education Commission (No. HJW-R-2019-66-19). Clinical Pharmacy Key Specialty Construction Project of Shanghai (No. YZ2 017/5). Scientific research project of Science and Technology Commission of Shanghai Municipality (No. 18DZ1910604, No.19XD1400900).

\section{SUPPLEMENTARY MATERIAL}

The Supplementary Material for this article can be found online at: https://www.frontiersin.org/articles/10.3389/fphar.2021.668952/ full\#supplementary-material

Anderson, B. J., and Holford, N. H. (2008). Mechanism-based Concepts of Size and Maturity in Pharmacokinetics. Annu. Rev. Pharmacol. Toxicol. 48, 303-332. doi:10.1146/annurev.pharmtox.48.113006.094708

Chen, X., Wang, D. D., Xu, H., and Li, Z. P. (2020). Initial Dose Recommendation for Sirolimus in Paediatric Kaposiform Haemangioendothelioma Patients Based on Population Pharmacokinetics and Pharmacogenomics. J. Int. Med. Res. 48 (8), 300060520947627. doi:10.1177/0300060520947627

Cheng, X., Zhao, Y., Gu, H., Zhao, L., Zang, Y., Wang, X., et al. (2020). The First Study in Pediatric: Population Pharmacokinetics of Sirolimus and its Application in Chinese Children with Immune Cytopenia. Int. J. Immunopathol Pharmacol. 34, 2058738420934936. doi:10.1177/2058738420934936 
Cloughesy, T. F., Yoshimoto, K., Nghiemphu, P., Brown, K., Dang, J., Zhu, S., et al. (2008). Antitumor Activity of Rapamycin in a Phase I Trial for Patients with Recurrent PTEN-Deficient Glioblastoma. Plos Med. 5 (1), e8. doi:10.1371/ journal.pmed.0050008

Euvrard, S., Morelon, E., Rostaing, L., Goffin, E., Brocard, A., Tromme, I., et al. (2012). Sirolimus and Secondary Skin-Cancer Prevention in Kidney Transplantation. N. Engl. J. Med. 367 (4), 329-339. doi:10.1056/ NEJMoa1204166

Hammill, A. M., Wentzel, M., Gupta, A., Nelson, S., Lucky, A., Elluru, R., et al. (2011). Sirolimus for the Treatment of Complicated Vascular Anomalies in Children. Pediatr. Blood Cancer 57 (6), 1018-1024. doi:10.1002/pbc.23124

Hao, G. X., Huang, X., Zhang, D. F., Zheng, Y., Shi, H. Y., Li, Y., et al. (2018). Population Pharmacokinetics of Tacrolimus in Children with Nephrotic Syndrome. Br. J. Clin. Pharmacol. 84 (8), 1748-1756. doi:10.1111/bcp.13605

Huber, S., Bruns, C. J., Schmid, G., Hermann, P. C., Conrad, C., Niess, H., et al. (2007). Inhibition of the Mammalian Target of Rapamycin Impedes Lymphangiogenesis. Kidney Int. 71 (8), 771-777. doi:10.1038/sj.ki.5002112

Ji, Y., Chen, S., Xiang, B., Li, K., Xu, Z., Yao, W., et al. (2017). Sirolimus for the Treatment of Progressive Kaposiform Hemangioendothelioma: A Multicenter Retrospective Study. Int. J. Cancer 141 (4), 848-855. doi:10.1002/ijc.30775

Klümpen, H. J., Beijnen, J. H., Gurney, H., and Schellens, J. H. (2010). Inhibitors of mTOR. Oncologist 15 (12), 1262-1269. doi:10.1634/theoncologist.2010-0196

Kobayashi, S., Kishimoto, T., Kamata, S., Otsuka, M., Miyazaki, M., and Ishikura, H. (2007). Rapamycin, a Specific Inhibitor of the Mammalian Target of Rapamycin, Suppresses Lymphangiogenesis and Lymphatic Metastasis. Cancer Sci. 98 (5), 726-733. doi:10.1111/j.1349-7006.2007.00439.x

Komiya, T., Memmott, R. M., Blumenthal, G. M., Bernstein, W., Ballas, M. S., De Chowdhury, R., et al. (2019). A Phase I/II Study of Pemetrexed with Sirolimus in Advanced, Previously Treated Non-small Cell Lung Cancer. Transl Lung Cancer Res. 8 (3), 247-257. doi:10.21037/tlcr.2019.04.19

Laforgia, N., Schettini, F., De Mattia, D., Martinelli, D., Ladisa, G., and Favia, V. (2016). Lymphatic Malformation in Newborns as the First Sign of Diffuse Lymphangiomatosis: Successful Treatment with Sirolimus. Neonatology 109 (1), 52-55. doi:10.1159/000440939

Lagrèze, W. A., Joachimsen, L., Gross, N., Taschner, C., and Rössler, J. (2019). Sirolimus-induced Regression of a Large Orbital Lymphangioma. Orbit 38 (1), 79-80. doi:10.1080/01676830.2018.1436569

Lee, D. F., and Hung, M. C. (2007). All Roads lead to mTOR: Integrating Inflammation and Tumor Angiogenesis. Cell Cycle 6 (24), 3011-3014. doi:10.4161/cc.6.24.5085

Miceli, A., and Stewart, K. M. (2020). "Lymphangioma," in StatPearls (Treasure Island.

Mizuno, T., Fukuda, T., Christians, U., Perentesis, J. P., Fouladi, M., and Vinks, A. A. (2017a). Population Pharmacokinetics of Temsirolimus and Sirolimus in Children with Recurrent Solid Tumours: a Report from the Children's Oncology Group. Br. J. Clin. Pharmacol. 83 (5), 1097-1107. doi:10.1111/ bcp. 13181

Mizuno, T., Fukuda, T., Emoto, C., Mobberley-Schuman, P. S., Hammill, A. M., Adams, D. M., et al. (2017b). Developmental Pharmacokinetics of Sirolimus: Implications for Precision Dosing in Neonates and Infants with Complicated Vascular Anomalies. Pediatr. Blood Cancer 64 (8). doi:10.1002/pbc.26470

Mussin, N., Oh, S. C., Lee, K. W., Park, M. Y., Seo, S., Yi, N. J., et al. (2017). Sirolimus and Metformin Synergistically Inhibits Colon Cancer In Vitro and In Vivo. J. Korean Med. Sci. 32 (9), 1385-1395. doi:10.3346/ jkms.2017.32.9.1385

Neuhaus, P., Klupp, J., and Langrehr, J. M. (2001). mTOR Inhibitors: an Overview. Liver Transpl. 7 (6), 473-484. doi:10.1053/jlts.2001.24645

Niu, W. J., Sun, T., Liu, L., Liu, X. Q., Zhang, R. F., Yin, L., et al. (2019). Population Pharmacokinetics and Dosing Regimen Optimisation of Lopinavir in Chinese Adults Infected with HIV. Basic Clin. Pharmacol. Toxicol. 124 (4), 456-465. doi:10.1111/bcpt.13154

Pinto-Leite, R., Botelho, P., Ribeiro, E., Oliveira, P. A., and Santos, L. (2009). Effect of Sirolimus on Urinary Bladder Cancer T24 Cell Line. J. Exp. Clin. Cancer Res. 28, 3. doi:10.1186/1756-9966-28-3

Reinglas, J., Ramphal, R., and Bromwich, M. (2011). The Successful Management of Diffuse Lymphangiomatosis Using Sirolimus: a Case Report. Laryngoscope 121 (9), 1851-1854. doi:10.1002/lary.21927

Shibuya, M. (2011). Vascular Endothelial Growth Factor (VEGF) and its Receptor (VEGFR) Signaling in Angiogenesis: A Crucial Target for Anti- and Proangiogenic Therapies. Genes Cancer 2 (12), 1097-1105. doi:10.1177/ 1947601911423031

Tamashiro, E. Y., Felipe, C. R., Genvigir, F. D. V., Rodrigues, A. C., Campos, A. B., Hirata, R. D. C., et al. (2017). Influence of CYP3A4 and CYP3A5 Polymorphisms on Tacrolimus and Sirolimus Exposure in Stable Kidney Transplant Recipients. Drug Metab. Pers Ther. 32 (2), 89-95. doi:10.1515/dmpt-2016-0036

Wan, X., and Helman, L. J. (2007). The Biology behind mTOR Inhibition in Sarcoma. Oncologist 12 (8), 1007-1018. doi:10.1634/theoncologist.12-8-1007

Wang, D. D., Chen, X., Xu, H., and Li, Z. P. (2020). Initial Dosage Recommendation for Sirolimus in Children with Tuberous Sclerosis Complex. Front. Pharmacol. 11, 890. doi:10.3389/fphar.2020.00890

Yi, Z., Liu, B., Sun, X., Rong, G., Wang, W., Li, H., et al. (2020). Safety and Efficacy of Sirolimus Combined with Endocrine Therapy in Patients with Advanced Hormone Receptor-Positive Breast Cancer and the Exploration of Biomarkers. Breast 52, 17-22. doi:10.1016/j.breast.2020.04.004

Zhang, J., Dai, Y., Liu, Z., Zhang, M., Li, C., Chen, D., et al. (2017). Effect of CYP3A4 and CYP3A5 Genetic Polymorphisms on the Pharmacokinetics of Sirolimus in Healthy Chinese Volunteers. Ther. Drug Monit. 39 (4), 406-411. doi:10.1097/FTD.0000000000000415

Conflict of Interest: The authors declare that the research was conducted in the absence of any commercial or financial relationships that could be construed as a potential conflict of interest.

Publisher's Note: All claims expressed in this article are solely those of the authors and do not necessarily represent those of their affiliated organizations, or those of the publisher, the editors and the reviewers. Any product that may be evaluated in this article, or claim that may be made by its manufacturer, is not guaranteed or endorsed by the publisher.

Copyright (c) 2021 Chen, Wang, Wang, Huang, Yu, Lu, Zhai, Xu and Li. This is an open-access article distributed under the terms of the Creative Commons Attribution License (CC BY). The use, distribution or reproduction in other forums is permitted, provided the original author(s) and the copyright owner(s) are credited and that the original publication in this journal is cited, in accordance with accepted academic practice. No use, distribution or reproduction is permitted which does not comply with these terms. 